\title{
A Flexible Approach towards trans-Fused Polycyclic Tetrahydropyrans. A Synthesis of Prymnesin and Yessotoxin Units
}

\author{
Barry M. Trost ${ }^{*}$ and Young Ho Rhee \\ Department of Chemistry \\ Stanford University \\ Stanford, CA 94305-5080
}

Supporting Information: Experimental Procedure for the preparation of compounds 3 , 5, $7-17$, and acetate derivatives of compounds 7 and 8 .

General. All reactions were run under an atmosphere of dry Nitrogen unless otherwise indicated. Anhydrous solvents were transferred by oven-dried syringe or canula. Glass equipment was flame dried under a stream of nitrogen. Dichloromethane $\left(\mathrm{CH}_{2} \mathrm{Cl}_{2}\right)$ was distilled from calcium hydride. Tetrahydrofuran (THF) and diethyl ether were distilled from sodium benzophenone ketyl. Melting points were determined on a Thomas-Hoover melting point apparatus in open capillaries and are uncorrected. Optical rotations were determined using a JASCO DIP-360 in 50mm cells. Flash chromatography employed E.Merck silica gel (Kieselgel 60, 230-400 mesh). Analytical TLC was performed on Merck glass plates coated with silica using UV light, $1 \% \mathrm{KMnO}_{4}$ in water, or $0.25 \% \mathrm{p}$ anisaldehyde in ethanol for visualization. Infrared spectra were recorded on a Perkin Elmer FT-IR Paragon 500. ${ }^{1} \mathrm{H}$ and ${ }^{13} \mathrm{C}$ NMR spectra were reported using a Varian Gemini 300 (300 MHz, $75 \mathrm{MHz}$ ) and Gemini 500. Microanalyses were performed by MH-W Laboratories, Phoenix, AZ. Mass spectra were provided by the Mass Spectrometry Facility at the University of California-San Francisco.

\section{Preparation of (2R, 3S)-3-Benzyloxy-hex-5-yne-1,2-diol (3)}

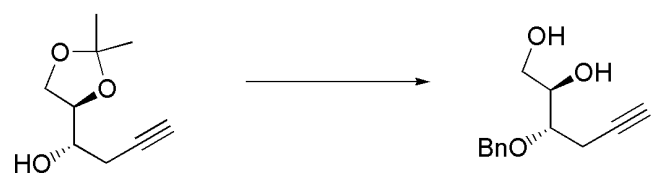


To a stirred solution of $(\mathbf{2} \boldsymbol{R}, \mathbf{3 S})$-1,2-O-Isopropylidenehex-5-yne-1,2,3-triol (170 mg, $0.998 \mathrm{mmol})^{1}$ in anhydrous DMF $(2 \mathrm{~mL})$ was added sodium hydride $(40 \mathrm{mg}, 60 \%$ dispersion in mineral oil, $0.998 \mathrm{mmol}$ ) in one portion at $0^{\circ} \mathrm{C}$. After stirring for $30 \mathrm{~min}$, neat benzyl bromide $(256 \mathrm{mg}, 1.50 \mathrm{mmmol})$ was added at $0^{\circ} \mathrm{C}$ and the reaction mixture was stirred for $1 \mathrm{~h}$. The solution was poured onto water:ice mixture $(5 \mathrm{~g})$. The aqueous layer was extracted with ether $\left(3 \_30 \mathrm{~mL}\right)$. The organic layers were combined, dried over $\mathrm{MgSO}_{4}$ and concentrated in vacuo. The residual oil was purified by column chromatography (silica gel, eluted with pet-ether:EtOAc $=15: 1$ ) to give the title compound as a clear oil (216 mg, 0.830 mmol, $83 \%$ yield), $R_{\mathrm{f}} 0.60$ (petether:EtOAc=9:1). $[\alpha]_{\mathrm{D}} 68.7\left(c 0.17, \mathrm{CHCl}_{3}\right)$.

IR (neat film): $v$ 3293, 2988, 2883, 1955, 1455, 1371, 1255, 1212, $1074 \mathrm{~cm}^{-1} .{ }^{1} \mathrm{H}$ NMR $\left(\mathrm{CDCl}_{3}, 300 \mathrm{MHz}\right): \delta 7.35(\mathrm{~m}, 5 \mathrm{H}), 4.79(\mathrm{~d}, \mathrm{~J}=11.4 \mathrm{~Hz}, 1 \mathrm{H}), 4.59(\mathrm{~d}, \mathrm{~J}=11.4 \mathrm{~Hz}, 1 \mathrm{H})$, $4.20(\mathrm{~m}, 1 \mathrm{H}), 4.08(\mathrm{dd}, \mathrm{J}=8.7,6.6 \mathrm{~Hz}, 1 \mathrm{H}), 3.90(\mathrm{dd}, \mathrm{J}=8.7,5.4 \mathrm{~Hz}, 1 \mathrm{H}), 3.56(\mathrm{~m}, 1 \mathrm{H})$, $2.65(\mathrm{ddd}, \mathrm{J}=17.1,4.5,2.7 \mathrm{~Hz}, 1 \mathrm{H}), 2.52(\mathrm{ddd}, \mathrm{J}=16.8,4.5,2.7 \mathrm{~Hz}, 1 \mathrm{H}), 2.04(\mathrm{t}, \mathrm{J}=2.7$ $\mathrm{Hz}, 1 \mathrm{H}), 1.40$ (s, $3 \mathrm{H}), 1.35$ (s, 3H). ${ }^{13} \mathrm{C} \mathrm{NMR}\left(\mathrm{CDCl}_{3}, 75 \mathrm{MHz}\right): \delta 137.9,128.4,127.9$, 127.8, 109.3, 80.5, 77.5, 76.4, 72.4, 70.4, 66.6, 26.7, 25.2, 21.0. Anal. Calcd for $\mathrm{C}_{16} \mathrm{H}_{20} \mathrm{O}_{3}: \mathrm{C}, 73.82 ; \mathrm{H}, 7.74$. Found: $\mathrm{C}, 73.66 ; \mathrm{H}, 7.88$.

A mixture of compound this compound $(196 \mathrm{mg}, 0.753 \mathrm{mmol})$ and p-toluenesulfonic acid $(10 \mathrm{mg})$ in anhydrous $\mathrm{MeOH}(10 \mathrm{~mL})$ was stirred under reflux for $4 \mathrm{~h}$. The reaction mixture was cooled to rt and $\mathrm{MeOH}$ was removed in vacuo. The residual oil was purified by column chromatography (silica gel, eluted with pet-ether:EtOAc $=1: 1$ ) to give the title compound as a clear oil $\left(149 \mathrm{mg}, 0.676 \mathrm{mmol}, 90 \%\right.$ yield), $\mathrm{R}_{\mathrm{f}} 0.20$ (pet-ether:EtOAc $=$ $1: 1)$. $[\alpha]_{\mathrm{D}} 46.2\left(\mathrm{c} 0.36, \mathrm{CHCl}_{3}\right)$.

IR (neat film): v 3295, 2925, 1455, 1345, 1076 $\mathrm{cm}^{-1} .{ }^{1} \mathrm{H}$ NMR $\left(\mathrm{CDCl}_{3}, 300 \mathrm{MHz}\right): \delta 7.34$ (m, 5H), $4.73(\mathrm{~d}, \mathrm{~J}=11.5 \mathrm{~Hz}, 1 \mathrm{H}), 4.53(\mathrm{~d}, \mathrm{~J}=11.5 \mathrm{~Hz}, 1 \mathrm{H}), 3.81(\mathrm{~m}, 1 \mathrm{H}), 3.62(\mathrm{~m}, 3 \mathrm{H})$, 3.12 (br s, 1H), $2.57(\mathrm{~m}, 2 \mathrm{H}), 2.04(\mathrm{t}, \mathrm{J}=2.4 \mathrm{~Hz}, 1 \mathrm{H}) .{ }^{13} \mathrm{C} \mathrm{NMR}\left(\mathrm{CDCl}_{3}, 75 \mathrm{MHz}\right): \delta$ 137.6, 128.4, 127.9, 127.8, 80.6, 77.8, 72.3, 72.2, 70.5, 63.2, 20.3. Anal. Calcd for $\mathrm{C}_{13} \mathrm{H}_{16} \mathrm{O}_{3}: \mathrm{C}, 70.89 ; \mathrm{H}, 7.32$. Found: $\mathrm{C}, 71.20 ; \mathrm{H}, 7.44$.

\footnotetext{
${ }^{1}$ Peng, Z.-H.; Li, Y.-L., Wu, W.-L., Liu, C.-X., Wu, Y.-L. J.Chem.Soc., Perkin Trans 1. 1996, 1057.
} 


\section{Preparation of (3-Benzyloxy-3,4-dihydro-2H-pyran-2-yl)-methanol (5a)}

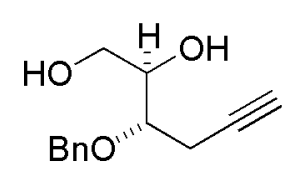

3
$\mathrm{CpRu}\left(\mathrm{PR}_{3}\right)_{2} \mathrm{Cl}(4)$

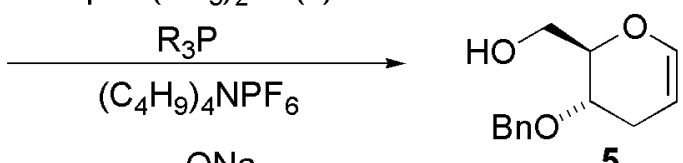

$\mathrm{ONa}$<smiles>O=C1CCC(=O)N1</smiles>

DMF, $85^{\circ}$

$70 \%$

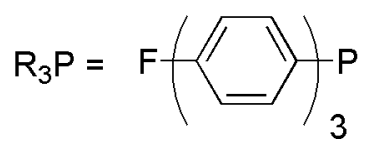

A mixture of compound 3 (131 mg, $0.596 \mathrm{mmol}$ ), Ru complex (37 mg, $0.044 \mathrm{mmol}$ ), ligand (56 mg, $0.177 \mathrm{mmol})$, N-Hydroxysuccinimide sodium salt (81 mg, $0.596 \mathrm{mmol}$ ), and tetra-n-butylammonium hexafluorophosphate $(52 \mathrm{mg}, 0.134 \mathrm{mmol})$ in DMF $(1.4 \mathrm{~mL}$, $0.4 \mathrm{M})$ was placed in a pre-heated oil bath at $85^{\circ} \mathrm{C}$ and stirred for $26 \mathrm{~h}$. The reaction mixture was cooled to rt and DMF was removed under reduced pressure. The residual oil was diluted with EtOAc $(20 \mathrm{~mL})$ and washed with water $\left(2 \_5 \mathrm{~mL}\right)$. The organic layer was separated and the aqueous layer was extracted with EtOAc $(3 \ldots 20 \mathrm{~mL})$. The organic layers were combined, dried over anh. $\mathrm{MgSO}_{4}$ and concentrated in vacuo. The residual oil was purified by column chromatography (florisil, eluted with pet-ether:EtOAc $=10: 1$ ) to provide the title compound (92 $\mathrm{mg}, 0.104 \mathrm{mmol}, 70 \%$ yield) as a clear oil, $\mathrm{R}_{\mathrm{f}} 0.85$ (petether:EtOAc=1:3). $[\alpha]_{\mathrm{D}} 89.9\left(\mathrm{c} 1.80, \mathrm{CHCl}_{3}\right)$.

IR (neat film): v 3440, 3064, 3031, 2922, 1958, 1656, 1589, 1496, 1454, 1239, $1078 \mathrm{~cm}^{-}$ 1. ${ }^{1} \mathrm{H}$ NMR $\left(\mathrm{CDCl}_{3}, 300 \mathrm{MHz}\right): \delta 7.30$ - $7.40(\mathrm{~m}, 5 \mathrm{H}), 6.33(\mathrm{~m}, 1 \mathrm{H}), 4.70(\mathrm{~d}, \mathrm{~J}=11.7 \mathrm{~Hz}$, 1H), $4.66(\mathrm{~d}, \mathrm{~J}=11.7 \mathrm{~Hz}, 1 \mathrm{H}), 4.56(\mathrm{~m}, 1 \mathrm{H}), 3.88(\mathrm{~m}, 2 \mathrm{H}), 3.65(\mathrm{~m}, 2 \mathrm{H}), 2.45(\mathrm{~m}, 1 \mathrm{H})$, $2.15(\mathrm{~m}, 1 \mathrm{H}), 2.08(\mathrm{~m}, 1 \mathrm{H}) .{ }^{13} \mathrm{C} \mathrm{NMR}\left(\mathrm{CDCl}_{3}, 75 \mathrm{MHz}\right): \delta 142.9,138.0,128.5,127.9$, 127.8, 98.0, 86.0, 71.1, 71.0, 62.4, 26.8. Anal. Calcd for $\mathrm{C}_{13} \mathrm{H}_{16} \mathrm{O}_{3}: \mathrm{C}, 70.88 ; \mathrm{H}, 7.32$. Found: C, 71.00; H 7.30.

\section{Preparation of 4,6-di- $O$-Benzyl-3-deoxy-D-glucal (5b)}<smiles>OC[C@H]1OC=CC[C@@H]1Br</smiles><smiles>BrOC[C@H]1OC=CC[C@@H]1Br</smiles> 
To a stirred solution of alcohol $\mathbf{5 a}(110 \mathrm{mg}, 0.501 \mathrm{mmol})$ in dry DMF $(1 \mathrm{~mL})$ at $0^{\circ} \mathrm{C}$ was added sodium hydride ( $22 \mathrm{mg}, 0.558 \mathrm{mmol}, 60 \%$ oil dispersion) and the solution was stirred for $30 \mathrm{~min}$. Neat benzyl bromide $(171 \mathrm{mg}, 1.00 \mathrm{mmol}$ ) was added dropwise to this solution at $0^{\circ} \mathrm{C}$ and the solution was stirred for $4 \mathrm{~h}$. The reaction mixture was then poured into a water:ice mixture $(5 \mathrm{~g})$ and vigorously stirred for $10 \mathrm{~min}$. The aqueous layer was extracted with ether $\left(2_{-}-30 \mathrm{~mL}\right)$. The organic layers were combined, dried over anh. $\mathrm{Na}_{2} \mathrm{SO}_{4}$ and concentrated in vacuo. The residual oil was purified by column chromatography (silica gel, eluted with pet-ether;EtOAc $=4: 1$ ) to give the title compound as a colorless oil (120 mg, $0.387 \mathrm{mmol}, 77 \%$ yield), $\mathrm{R}_{\mathrm{f}} 0.75$ (pet-ether:EtOAc $=10: 1$ ). $[\alpha]_{\mathrm{D}}+95.3\left(c 0.66, \mathrm{CHCl}_{3}\right)$. The spectral data are in full accord with literature values. ${ }^{2}$ IR (neat film): $v$ 3064, 3031, 2866, 1655, 1496, 1454, $1364 \mathrm{~cm}^{-1} .{ }^{1} \mathrm{H} \mathrm{NMR}\left(\mathrm{CDCl}_{3}\right.$, 300MHz): $\delta 7.20-7.30(\mathrm{~m}, 10 \mathrm{H}), 6.37(\mathrm{~d}, \mathrm{~J}=6.1 \mathrm{~Hz}, 1 \mathrm{H}), 4.66(\mathrm{~d}, \mathrm{~J}=11.7 \mathrm{~Hz}, 1 \mathrm{H})$, $4.62(\mathrm{~m}, 1 \mathrm{H}), 4.60(\mathrm{~m}, 2 \mathrm{H}), 4.53(\mathrm{~d}, \mathrm{~J}=11.7 \mathrm{~Hz}, 1 \mathrm{H}), 3.72$ - $4.00(\mathrm{~m}, 4 \mathrm{H}), 2.40(\mathrm{~m}, 1 \mathrm{H})$, $2.12(\mathrm{~m}, 1 \mathrm{H}) .{ }^{13} \mathrm{C} \mathrm{NMR}\left(\mathrm{CDCl}_{3}, 75 \mathrm{MHz}\right): \delta 143.2,138.3,138.2,128.4,127.8,127.7$, $127.6,97.1,76.8,73.5,71.1,70.5,69.0,26.5$.

\section{Preparation of $(2 S, 3 R, 5 S, 6 R)-5$-Benzyloxy-6-benzyloxymethyl-2-propynyl- tetrahydro-pyran-3-ol (7 / 8)}

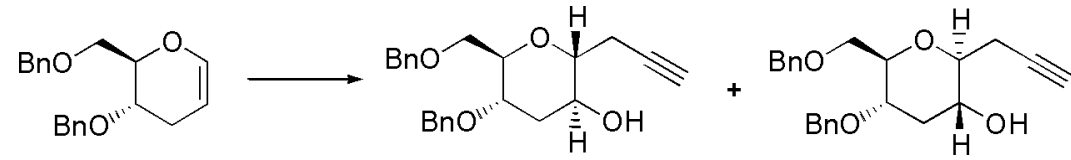

To a stirred solution of compound $\mathbf{5 b}(670 \mathrm{mg}, 2.16 \mathrm{mmol})$ in $\mathrm{CH}_{2} \mathrm{Cl}_{2}(20 \mathrm{~mL})$ was added freshly prepared dimethyldioxirane ${ }^{3}(0.1 \mathrm{M}$ solution in acetone, $23 \mathrm{~mL})$ dropwise at -78 ${ }^{\circ} \mathrm{C}$ and the solution was stirred for $30 \mathrm{~min}$. The solution was concentrated under reduced pressure and the residual solvent was completely removed under high vacuum. The crude oil was dissolved in ether $(20 \mathrm{~mL})$ and cooled to $-78{ }^{\circ} \mathrm{C}$. To this solution was added freshly prepared allenylmagnesium bromide (1 $\mathrm{M}$ solution in ether, $3 \mathrm{~mL})$ and the resulting solution was stirred for $2 \mathrm{~h}$ at that temperature. Water $(10 \mathrm{~mL})$ and aqueous $1 \mathrm{~N}$ $\mathrm{HCl}$ solution $(10 \mathrm{~mL})$ was slowly added to this solution and the solution was allowed to

\footnotetext{
${ }^{2}$ Kaye, A.; Stephen, N.; Colin, R. Tetrahedron Lett. 1988, 32, 1841.

${ }^{3}$ Halcomb, R. L.; Danishefsky, S. J. J. Am. Chem. Soc. 1989, 111, 6661.
} 
warm to $0{ }^{\circ} \mathrm{C}$. The organic layer was separated and the aqueous layer was extracted with ether $(3 \times 20 \mathrm{~mL})$. The organic layers were combined, dried over anh. $\mathrm{MgSO}_{4}$ and concentrated in vacuo. The residual oil was purified by column chromatography (silica gel, pet-ether:EtOAc $=10: 1$ to $4: 1$ ) to give the title compound as an inseparable $4: 1$ mixture of diastereomers 7 / 8 (617 $\mathrm{mg}, 1.69 \mathrm{mmol}, 78 \%$ yield), which was used for the next reaction, $\mathrm{R}_{\mathrm{f}} 0.55$ (pet-ether : $\mathrm{EtOAc}=4: 1$ ).

IR (neat film): v 3440, 3293, 2925, 2866, 2360, 1655, 1496, 1454, $1364 \mathrm{~cm}^{-1} .{ }^{1} \mathrm{H}$ NMR $\left(\mathrm{CDCl}_{3}, 300 \mathrm{MHz}\right): \delta$ 7.20-7.35 (m, $\left.10 \mathrm{H}\right), 4.52-4.58$ (m, $\left.4 \mathrm{H}\right), 3.57-3.67$ (m, $\left.3 \mathrm{H}\right), 3.50$ (m, $1 \mathrm{H}), 3.43(\mathrm{~m}, 1 \mathrm{H}), 3.25(\mathrm{~m}, 1 \mathrm{H}), 2.55-2.63(\mathrm{~m}, 2 \mathrm{H}), 2.08$ (m, $1 \mathrm{H}), 2.06,1.98$ (1 : 4, t, $1 \mathrm{H}), 1.94(\mathrm{~m}, 2 \mathrm{H})$.

Preparation of $(2 S, 3 R, 5 S, 6 R)$-5-Benzyloxy-6-benzyloxymethyl-2-prop-ynyl-3acetoxytetrahydropyran and (7-acetate) $(2 R, 3 S, 5 S, 6 R)$-5-Benzyloxy-6benzyloxymethyl-2-prop-ynyl-3-acetoxytetrahydropyran (8-acetate)

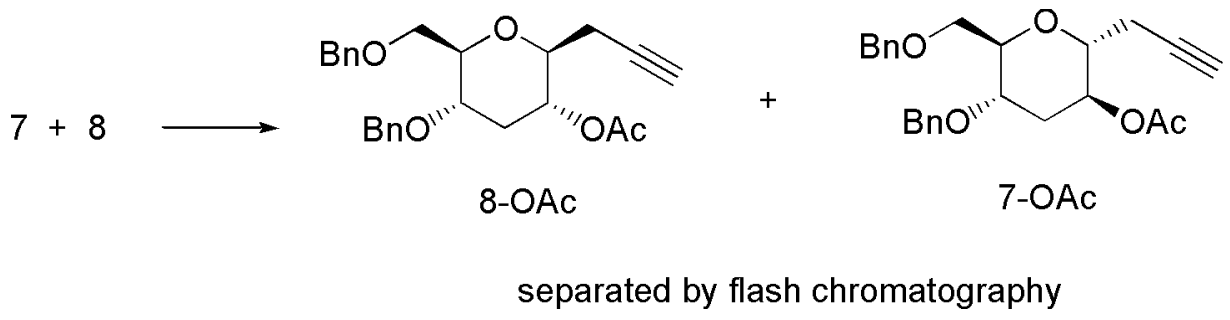

To a stirred solution of a comparable mixture of alcohols 7 / 8 ( $2: 1$ mixture, $37 \mathrm{mg}, 0.10$ mmol) in $\mathrm{CH}_{2} \mathrm{Cl}_{2}(1 \mathrm{~mL})$ was added triethylamine $(300 \mu \mathrm{g}, 0.30 \mathrm{mmol})$ and the solution was stirred at $0{ }^{\circ} \mathrm{C}$ for $30 \mathrm{~min}$. Neat acetic anhydride $(153 \mu \mathrm{g}, 0.15 \mathrm{mmol})$ was added to this solution and the reaction mixture was stirred for $30 \mathrm{~min}$ at that temperature. Water (1 $\mathrm{mL}$ ) was added and the organic layer was separated and the aqueous layer was extracted with ether $(3 \times 5 \mathrm{~mL})$. The organic layers were combined, dried over anh. $\mathrm{MgSO}_{4}$ and concentrated in vacuo. The residual oil was purified by column chromatography (silica gel, eluted with pet-ether:EtOAc $=20: 1)$ to give the title compound acetate of alcohol $(2 S$, $3 R, 5 S, 6 R$ ) - 5 - B e n zy lo x y - 6 - b e n z y lo x y m et h y l-2 - p rop-ynyl-3acetoxytetrahydropyran (acetate derivative of alcohol 8) as a clear oil (12 $\mathrm{mg}, 0.30$ 
mmol, $30 \%$ yield), $\mathrm{R}_{\mathrm{f}} 0.50$ (pet-ether : EtOAc $\left.=7: 1\right)$. $[\alpha]_{\mathrm{D}}+10.7\left(c 0.41, \mathrm{CHCl}_{3}\right)$. IR (neat film): v 3290, 2867, 1735, 1455, 1367, 1241, $1094 \mathrm{~cm}^{-1} .{ }^{1} \mathrm{H}$ NMR $\left(\mathrm{CDCl}_{3}\right.$, 500MHz): $\delta 7.25-7.35$ (m, $10 \mathrm{H}), 4.74$ (ddd, $J=11.4,9.6,4.6 \mathrm{~Hz}, 1 \mathrm{H}), 4.67$ (d, $J=12.3$ $\mathrm{Hz}, 1 \mathrm{H}), 4.61(\mathrm{~d}, J=12.3 \mathrm{~Hz}, 1 \mathrm{H}), 4.60(\mathrm{~d}, J=11.3 \mathrm{~Hz}, 1 \mathrm{H}), 4.42(\mathrm{~d}, J=11.3 \mathrm{~Hz}, 1 \mathrm{H})$, $3.82(\mathrm{dd}, J=11.0,1.8 \mathrm{~Hz}, 1 \mathrm{H}), 3.69(\mathrm{dd}, J=11.0,5.3 \mathrm{~Hz}, 1 \mathrm{H}), 3.58(\mathrm{ddd}, J=10.8,9.7$, $4.6 \mathrm{~Hz}, 1 \mathrm{H}), 3.52$ (td, $J=9.7,4.5 \mathrm{~Hz}, 1 \mathrm{H}), 3.49$ (ddd, $J=9.7,5.3,1.8 \mathrm{~Hz}, 1 \mathrm{H}), 2.73$ (ddd, $J=11.7,4.6,4.5 \mathrm{~Hz}, 1 \mathrm{H}$ ), 2.57 (ddd, $J=17.0,4.6,2.6 \mathrm{~Hz}, 1 \mathrm{H}$ ), 2.49 (ddd, $J=$ 17.0, 9.6, 2.6 Hz, $1 \mathrm{H}$ ), 2.09 (s, $3 \mathrm{H}$ ), 2.01 (t, $J=2.6 \mathrm{~Hz}, 1 \mathrm{H}$ ), 1.52 (app q, $J \sim 11 \mathrm{~Hz}, 1$ H). ${ }^{13} \mathrm{C} \mathrm{NMR}\left(\mathrm{CDCl}_{3}, 125 \mathrm{MHz}\right): \delta 170.0,138.4,137.8,128.4,128.3,127.8,127.7$, 127.5, 80.8, 80.1, 77.1, 73.4, 71.9, 71.3, 69.9, 69.8, 69.1, 34.9, 22.3, 21.1. HRMS Calcd for $\mathrm{C}_{18} \mathrm{H}_{21} \mathrm{O}_{5}\left(\mathrm{M}^{+}-\mathrm{C}_{6} \mathrm{H}_{5} \mathrm{CH}_{2}\right)$ : 317.1389. Found : 317.1383 .

Further elution provided $(2 R, 3 S, 5 S, 6 R)$-5-Benzyloxy-6-benzyloxymethyl-2-propynyl-3-acetoxytetrahydropyran (acetate of alcohol 7) as a clear oil (62\% yield, $25 \mathrm{mg}$, $0.62 \mathrm{mmol}), \mathrm{R}_{\mathrm{f}} 0.50$ (pet-ether : EtOAc $\left.=7: 1\right)$. $[\alpha]_{\mathrm{D}}+18.5\left(\mathrm{c} 1.21, \mathrm{CHCl}_{3}\right)$.

IR (neat film): v 2935, 2873, 1740, 1233, 1144, $1083 \mathrm{~cm}^{-1} .{ }^{1} \mathrm{H}$ NMR $\left(\mathrm{CDCl}_{3}, 500 \mathrm{MHz}\right)$ : $\delta 7.22 \sim 7.36$ (m, $10 \mathrm{H}), 5.13$ (app td, $J=4.0,3.6 \mathrm{~Hz}, 1 \mathrm{H}), 4.65$ (d, $J=12.1 \mathrm{~Hz}, 1 \mathrm{H}), 4.59$ (d, $J=11.5 \mathrm{~Hz}, 1 \mathrm{H}), 4.57$ (d, $J=12.1 \mathrm{~Hz}, 1 \mathrm{H}), 4.47$ (d, $J=11.5 \mathrm{~Hz}, 1 \mathrm{H}), 4.02$ (ddd, $J=$ 7.2, 6.9, $4.0 \mathrm{~Hz}, 1 \mathrm{H}), 3.82(\mathrm{~m}, 1 \mathrm{H}), 3.76(\mathrm{~m}, 1 \mathrm{H}), 3.72$ (m, $2 \mathrm{H}), 2.62$ (ddd, $J=16.8$, 7.2, $2.8 \mathrm{~Hz}, 1 \mathrm{H}$ ), 2.57 (ddd, $J=16.8,6.9,2.8 \mathrm{~Hz}, 1 \mathrm{H}$ ), 2.15 (ddd, $J=13.8,4.5,3.6 \mathrm{~Hz}$, $1 \mathrm{H}), 2.10(\mathrm{~s}, 3 \mathrm{H}), 2.07$ (t, $J=2.8 \mathrm{~Hz}, 1 \mathrm{H}), 1.99(\mathrm{ddd}, J=13.8,8.6,4.0 \mathrm{~Hz}, 1 \mathrm{H}) .{ }^{13} \mathrm{C}$ NMR $\left(\mathrm{CDCl}_{3}, 125 \mathrm{MHz}\right): \delta 170.2,138.2,138.0,128.4,128.3,127.7,127.6,79.5,73.6$, 73.4, 73.3, 71.0, 70.8, 70.5, 69.8, 69.2, 29.6, 21.3, 20.7. HRMS Calcd for $\mathrm{C}_{18} \mathrm{H}_{21} \mathrm{O}_{5}\left(\mathrm{M}^{+}-\right.$ $\left.\mathrm{C}_{6} \mathrm{H}_{5} \mathrm{CH}_{2}\right): 317.1389$. Found : 317.1380 .

\section{Preparation of pure $(2 S, 3 R, 5 S, 6 R)$-5-Benzyloxy-6-benzyloxymethyl-2-propynyl-} tetrahydropyran-3-ol (8)

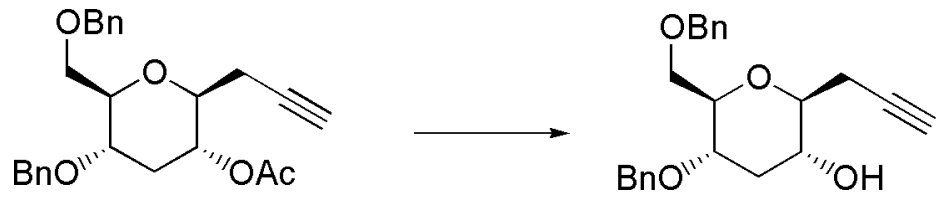


To a stirred solution of compound $(2 S, 3 R, 5 S, 6 R)-5$-Benzyloxy-6-benzyloxymethyl-2prop-ynyl-3-acetoxytetrahydropyran (acetate derivative of alcohol 8) (69 mg, 0.17 $\mathrm{mmol})$ in $\mathrm{MeOH}(1 \mathrm{~mL})$ and water $(0.3 \mathrm{~mL})$ was added potassium carbonate $(100 \mathrm{mg}$, $0.72 \mathrm{mmol}$ ) and the solution was stirred at $\mathrm{rt}$ for $3 \mathrm{~h}$. The solution was cooled to $\mathrm{rt}$ and diluted with ether $(5 \mathrm{~mL})$ and water $(1 \mathrm{~mL})$. The organic layers were combined, and the aqueous layer was extracted with ether $(3 \times 5 \mathrm{~mL})$. The organic layer was dried over anh. $\mathrm{MgSO}_{4}$, and concentrated in vacuo. The residual oil was purified by column chromatography ( as a clear oil (55 mg, $0.15 \mathrm{mmol}, 88 \%$ yield), $\mathrm{R}_{\mathrm{f}} 0.50$ (pet-ether : EtOAc $=4: 1$ ). $[\alpha]_{\mathrm{D}}$ $+34.1\left(\right.$ c $\left.1.2, \mathrm{CHCl}_{3}\right)$.

IR (neat film): 3440, 3294, 3030, 2929, 1496, 1454, 1384, $1096 \mathrm{~cm}^{-1} .{ }^{1} \mathrm{H} \mathrm{NMR}\left(\mathrm{CDCl}_{3}\right.$, 500MHz): $\delta 7.22-7.35(\mathrm{~m}, 10 \mathrm{H}), 4.64(\mathrm{~d}, J=11.3 \mathrm{~Hz}, 1 \mathrm{H}), 4.58(\mathrm{~d}, J=11.3 \mathrm{~Hz}, 1 \mathrm{H})$, $4.56(\mathrm{~d}, J=11.4 \mathrm{~Hz}, 1 \mathrm{H}), 4.43$ (d, $J=11.4 \mathrm{~Hz}, 1 \mathrm{H}), 3.77$ (dd, $J=11.0,2.0 \mathrm{~Hz}, 1 \mathrm{H})$, $3.67(\mathrm{dd}, J=11.0,5.0 \mathrm{~Hz}, 1 \mathrm{H}), 3.61(\mathrm{~m}, 1 \mathrm{H}), 3.50(\mathrm{ddd}, J=10.7,9.4,4.5 \mathrm{~Hz}, 1 \mathrm{H})$, 3.44 (ddd, $J=9.4,4.5,2.0 \mathrm{~Hz}, 1 \mathrm{H}), 3.29$ (m, $1 \mathrm{H}), 2.63$ (ddd, $J=5.5,5.4,2.7 \mathrm{~Hz}, 1 \mathrm{H})$, 2.56 (m, 2 H), 2.18 (br s, $1 \mathrm{H}), 2.06$ (t, $J=2.7 \mathrm{~Hz}, 1 \mathrm{H}), 1.49$ (app q, $J=10.7 \mathrm{~Hz}, 1 \mathrm{H})$. ${ }^{13} \mathrm{C} \mathrm{NMR}\left(\mathrm{CDCl}_{3}, 125 \mathrm{MHz}\right): \delta 138.4,138.0,128.4,128.3,127.8,127.7,127.5,80.6$, 80.5, 79.2, 73.4, 72.2, 71.1, 70.6, 69.2, 68.5, 38.1, 22.3. HRMS Calcd for $\mathrm{C}_{23} \mathrm{H}_{26} \mathrm{O}_{4}\left(\mathrm{M}^{+}\right)$: 366.1831. Found 366.1842.

Preparation of $(2 R, 3 S, 5 S, 6 R)$-5-Benzyloxy-6-benzyloxymethyl-2-prop-ynyltetrahydro-pyran-3-ol (7)

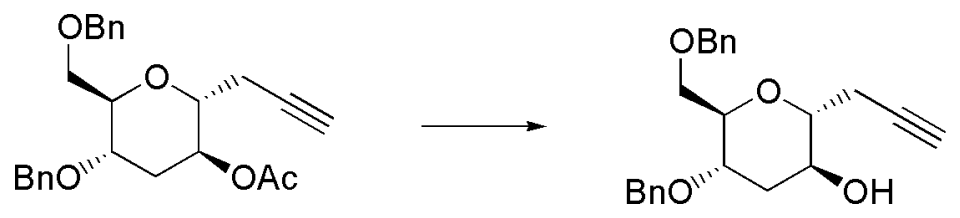

By using an analogous procedure, compound 7-acetate (22 $\mathrm{mg}, 0.054 \mathrm{mmol}$ ) was reacted with potassium carbonate $(14 \mathrm{mg}, 0.10 \mathrm{mmol})$ in $\mathrm{MeOH}(1 \mathrm{~mL})$ and water $(0.3 \mathrm{~mL})$ to give the title compound as a clear oil (13 mg, $0.040 \mathrm{mmol}, 75 \%$ yield), $\mathrm{R}_{\mathrm{f}} 0.50$ (pet-ether $: \mathrm{EtOAc}=4: 1) .[\alpha]_{\mathrm{D}}+46.3\left(\mathrm{c} 1.2, \mathrm{CHCl}_{3}\right)$. 
IR (neat film): 3292, 2926, 2360, 1454, 1363, $1094 \mathrm{~cm}^{-1} .{ }^{1} \mathrm{H} \mathrm{NMR}\left(\mathrm{CDCl}_{3}, 500 \mathrm{MHz}\right): \delta$ 7.20-7.35 (m, $10 \mathrm{H}), 4.57$ (d, $J=12.0 \mathrm{~Hz}, 1 \mathrm{H}), 4.55(\mathrm{~d}, J=12.0 \mathrm{~Hz}, 1 \mathrm{H}), 4.54$ (d, $J=$ $12.0 \mathrm{~Hz}, 1 \mathrm{H}), 4.48$ (d, J=12.0 Hz, $1 \mathrm{H}), 4.04(\mathrm{~m}, 1 \mathrm{H}), 3.87$ (m, $1 \mathrm{H}), 3.79$ (m, $2 \mathrm{H})$, 3.69 (dd, $J=10.4,5.2 \mathrm{~Hz}, 1 \mathrm{H}), 3.65$ (dd, $J=10.4,4.4 \mathrm{~Hz}, 1 \mathrm{H}$ ), 2.61 (ddd, $J=17.0,7.1$, $2.8 \mathrm{~Hz}, 1 \mathrm{H}$ ), 2.55 (ddd, $J=17.0,6.5,2.8 \mathrm{~Hz}, 1 \mathrm{H}), 1.98$ (t, $J=2.8 \mathrm{~Hz}, 1 \mathrm{H}), 1.94$ (m, 2 H). ${ }^{13} \mathrm{C} \mathrm{NMR}\left(\mathrm{CDCl}_{3}, 125 \mathrm{MHz}\right): \delta$ 138.1, 138.0, 128.4, 127.8, 127.7, 80.2, 75.4, 73.4, 73.3, 71.3, 70.8, 70.7, 68.9, 67.2, 32.4, 21.3. HRMS Calcd for $\mathrm{C}_{23} \mathrm{H}_{26} \mathrm{O}_{4}\left(\mathrm{M}^{+}\right)$: 366.1831 . Found : 366.1828 .

\section{Preparation of $(2 R, 5 S, 6 R)$-5-Benzyloxy-6-benzyloxymethyl-2-prop-2-ynyl-} tetrahydro-pyran-3-one (9):

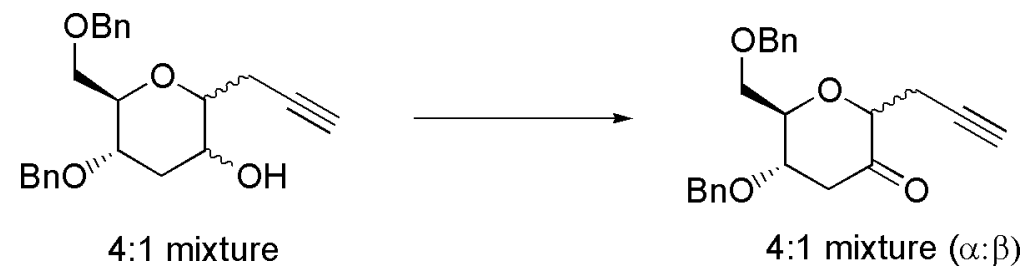

A solution of alcohol $7 / 8(150 \mathrm{mg}, 0.410 \mathrm{mmol}$, 4:1 mixture), pyridinium chlorochromate $(177 \mathrm{mg}, 0.820 \mathrm{mmol})$, sodium acetate $(25 \mathrm{mg})$ and well-ground $4 \mathrm{~A}$ molecular sieves $(150 \mathrm{mg})$ was stirred at $\mathrm{rt}$ for $5 \mathrm{~h}$. Diethyl ether $(30 \mathrm{~mL})$ was added to the reaction mixture and the resultant solution was stirred for $1 \mathrm{~h}$. The reaction mixture was filtered through a pad of celite and concentrated in vacuo. The residual oil was purified by column chromatography (silica gel, eluted with pet-ether : EtOAc $=10: 1$ ) to give a mixture of ketones (140 mg, $0.385 \mathrm{mmol}$ ) in a 4:1 mixture as a clear oil ( $94 \%$ yield). The spectral data for the major compound was obtained, $R_{f} 0.55$ (pet-ether : EtOAc $=4: 1$ ). IR (neat film): 3293, 2939, 2863, 1712, 1449, 1427, $1130 \mathrm{~cm}^{-1} .{ }^{1} \mathrm{H}$ NMR $\left(\mathrm{CDCl}_{3}\right.$, 500MHz): $\delta 7.10 \sim 7.22$ (m, $10 \mathrm{H}), 4.60$ (d, $J=12.2 \mathrm{~Hz}, 1 \mathrm{H}), 4.55(\mathrm{~d}, J=11.7 \mathrm{~Hz}, 1 \mathrm{H})$, $4.52(\mathrm{~d}, J=12.2 \mathrm{~Hz}, 1 \mathrm{H}), 4.42(\mathrm{~d}, J=11.7 \mathrm{~Hz}, 1 \mathrm{H}), 3.71(\mathrm{~m}, 2 \mathrm{H}), 4.31$ (dd, $J=9.0,4.4$ $\mathrm{Hz}, 1 \mathrm{H}), 4.19-4.16$ (m, $2 \mathrm{H}), 2.96$ (dd, $J=15.0,4.4 \mathrm{~Hz}, 1 \mathrm{H}), 2.80$ (ddd, $J=17.3,9.0$, $2.7 \mathrm{~Hz}, 1 \mathrm{H}), 2.70$ (dd, $J=15.0,6.1 \mathrm{~Hz}, 1 \mathrm{H}), 2.67$ (ddd, $J=17.3,4.4,2.7 \mathrm{~Hz}, 1 \mathrm{H}), 2.04$ (t, $J=2.7 \mathrm{~Hz}, 1 \mathrm{H}) \cdot{ }^{13} \mathrm{C} \mathrm{NMR}\left(\mathrm{CDCl}_{3}, 75 \mathrm{MHz}\right): \delta 208.5,137.8,137.3,128.5,128.4$, $127.9,127.8,127.7,127.7,78.5,78.9,74.3,73.7,73.5,70.8,70.4,70.3,41.5,19.8$. HRMS Calcd for $\mathrm{C}_{23} \mathrm{H}_{24} \mathrm{O}_{4}\left(\mathrm{M}^{+}\right)$: 364.1765. Found: 364.1769. 
Preparation of $(2 S, 5 S, 6 R)-5$-Benzyloxy-6-benzyloxymethyl-2-prop-2-ynyltetrahydro-pyran-3-one (10)

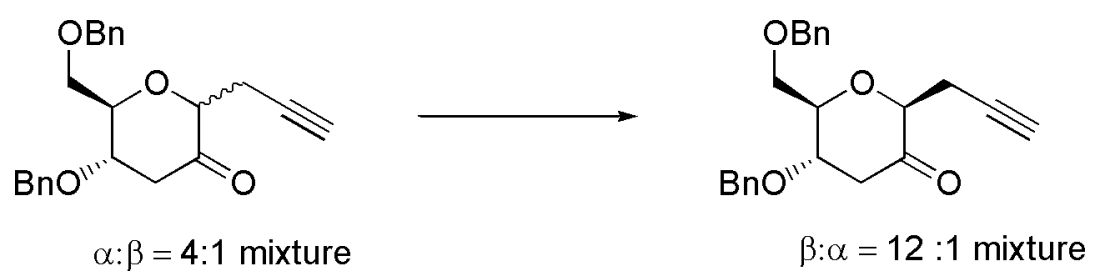

A solution of the ketone $9(\alpha: \beta=4: 1,147 \mathrm{mg}, 0.404 \mathrm{mmol})$ and diazabicycloundecene $(3.0 \mathrm{mg}, 0.02 \mathrm{mmol})$ in toluene $(5 \mathrm{~mL})$ was stirred at $\mathrm{rt}$ for $24 \mathrm{~h}$. The reaction mixture was washed with sat. $\mathrm{NH}_{4} \mathrm{Cl}$ solution $(2 \times 5 \mathrm{~mL})$. The organic layer was separated and the aqueous layer was extracted with ether $(2 \times 5 \mathrm{~mL})$. The organic layers were combined, dried over anh. $\mathrm{MgSO}_{4}$ and concentrated in vacuo. The residual oil was purified by column chromatography ( silica gel, eluted with pet-ether : EtOAc $=10: 1$ ) to give the title compound as a clear oil consisting of a $12: 1$ diastereomeric mixture (106 $\mathrm{mg}, 0.290 \mathrm{mmol}, 72 \%$ yield), $\mathrm{R}_{\mathrm{f}} 0.75$ (pet-ether : EtOAc $\left.=4: 1\right)$. [ $\left.\alpha\right]_{\mathrm{D}} 42.3$ (c 1.11, $\left.\mathrm{CHCl}_{3}\right)$.

IR (neat film): 3288, 2922, 1736, 1496, 1454, $1097 \mathrm{~cm}^{-1} .{ }^{1} \mathrm{H} \mathrm{NMR}\left(\mathrm{CDCl}_{3}, 500 \mathrm{MHz}\right): \delta$ 7.20-7.30 (m, $10 \mathrm{H}), 4.64(\mathrm{~d}, J=12.1 \mathrm{~Hz}, 1 \mathrm{H}), 4.59(\mathrm{~d}, J=12.1 \mathrm{~Hz}, 1 \mathrm{H}), 4.54(\mathrm{~d}, J=$ $11.7 \mathrm{~Hz}, 1 \mathrm{H}), 4.43(\mathrm{~d}, J=11.7 \mathrm{~Hz}, 1 \mathrm{H}), 4.07$ (dd, $J=5.2,4.4 \mathrm{~Hz}, 1 \mathrm{H}), 4.05$ (dd, $J=$ 6.7, $4.3 \mathrm{~Hz}, 1 \mathrm{H}), 3.97(\mathrm{dd}, J=4.6,4.4 \mathrm{~Hz}, 1 \mathrm{H}), 3.72(\mathrm{dd}, J=10.5,4.6 \mathrm{~Hz}, 1 \mathrm{H}), 3.69$ $(\mathrm{dd}, J=10.5,4.4 \mathrm{~Hz}, 1 \mathrm{H}), 2.90(\mathrm{dd}, J=15.4,4.4 \mathrm{~Hz}, 1 \mathrm{H}), 2.76(\mathrm{ddd}, J=17.1,4.3,2.7$ $\mathrm{Hz}, 1 \mathrm{H}), 2.66$ (dd, $J=15.4,5.2 \mathrm{~Hz}, 1 \mathrm{H}), 2.59$ (ddd, $J=17.1,6.7,2.7 \mathrm{~Hz}, 1 \mathrm{H}), 1.98(\mathrm{t}, J$ $=2.4 \mathrm{~Hz}, 1 \mathrm{H}) .{ }^{13} \mathrm{C} \mathrm{NMR}\left(\mathrm{CDCl}_{3}, 125 \mathrm{MHz}\right): \delta 207.4,137.9,137.3,128.5,128.4,127.9$, $127.8,127.7,127.7,79.9,79.6,79.5,74.1,73.6,70.7,69.9,69.7,41.6,20.4$. HRMS Calcd for $\mathrm{C}_{23} \mathrm{H}_{24} \mathrm{O}_{4}\left(\mathrm{M}^{+}\right)$: 364.1765. Found: 364.1757 . 
Preparation of 8 via the reduction of ketone 10:

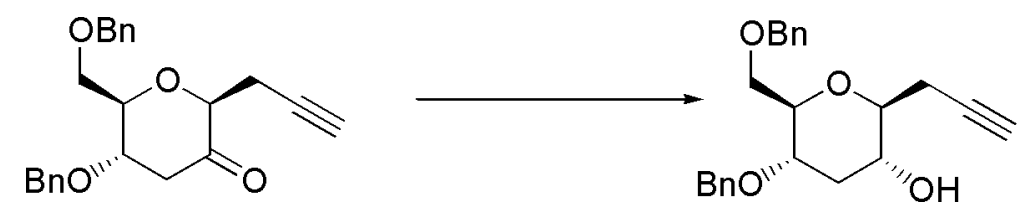

To a solution of ketone $10(130 \mathrm{mg}, 0.357 \mathrm{mmol})$ in THF $(5 \mathrm{~mL})$ was added lithium aluminum hydride $(13 \mathrm{mg}, 0.36 \mathrm{mmol})$ in one portion at $0^{\circ} \mathrm{C}$ and the reaction mixture was stirred for $1 \mathrm{~h}$. Sodium sulfate (decahydrate, $300 \mathrm{mg}$ ) was added slowly to this solution and the reaction mixture was stirred for an additional $30 \mathrm{~min}$. The solution was filtered through a pad of celite and concentrated in vacuo. The residual oil was purified by column chromatography ( silica gel, eluted with pet-ether : EtOAc $=10: 1$ ) to give the title compound as a clear oil (123 mg, $0.336 \mathrm{mmol}, 95 \%$ yield, $9: 1$ mixture). The spectral data of the major compound are in full accord with those previously obtained.

\section{One Pot-Epimerization and Reduction of 9 to Alcohol 8:}

To a stirred solution of ketone 9 (105 $\mathrm{mg}, 0.288 \mathrm{mmol}, 4: 1 \mathrm{mixture})$ in THF (2 mL) was added diazabicycloundecene $(2.2 \mathrm{mg}, 0.015 \mathrm{mmol})$ and the solution was stirred at $\mathrm{rt}$ for $24 \mathrm{~h}$. The reaction mixture was cooled to $0^{\circ} \mathrm{C}$ and lithium aluminum hydride $(11 \mathrm{mg}$, $0.288 \mathrm{mmol}$ ) was slowly added, then the solution was stirred at $0^{\circ} \mathrm{C}$ for $1 \mathrm{~h}$. Sodium sulfate decahydrate $(50 \mathrm{mg})$ was added slowly to this solution and the resulting solution was stirred for an additional $30 \mathrm{~min}$. The solution was filtered through a pad of celite and concentrated in vacuo. The residual oil was purified by column chromatography (silica gel, eluted with pet-ether : EtOAc $=10: 1)$ to give the title compound as a clear oil (62 $\mathrm{mg}, 0.178 \mathrm{mmol}, 62 \%$ yield, as 9:1 mixture). The spectral data are in full accord with those previously obtained.

Preparation of $(2 R, 3 S, 4 a R, 8 \mathrm{a} S)$-3-Benzyloxy-2-benzyloxymethyl-2,3,4,4a,8,8ahexahydro-pyrano[3,2-b]pyran (11) 

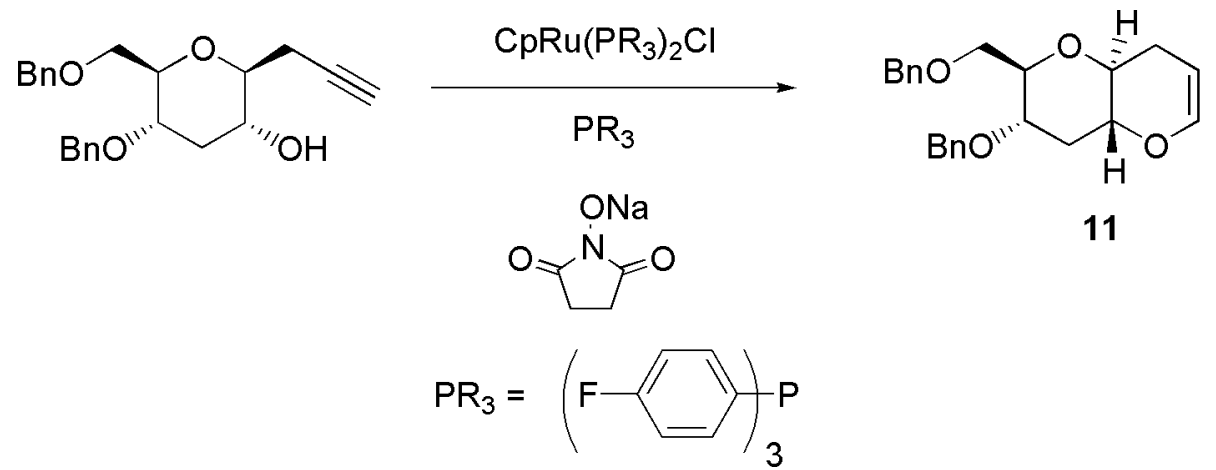

11

Ru complex 4a (12.4 mg, 0.0149 mmol), tris-p-fluorophenylphosphine $(18.8 \mathrm{mg}, 0.0596$ mmol), N-hydroxysuccinimide sodium salt (20.6 $\mathrm{mg}, 0.149 \mathrm{mmmol}$, pre-generated from N-hydroxysuccinimide and sodium hydride and stored in a dessicator), and tetra-nbutylammonium hexafluorophosphate $(17.3 \mathrm{mg}, 0.0447 \mathrm{mmol})$ was placed in $5 \mathrm{~mL}$ test tube. A solution of alcohol 8 (109 $\mathrm{mg}, 0.298 \mathrm{mmol})$ in DMF $(0.75 \mathrm{~mL}, 0.4 \mathrm{M})$ was added and the mixture was stirred at $85{ }^{\circ} \mathrm{C}$ for $26 \mathrm{~h}$. The reaction mixture was cooled to rt. Ether $(20 \mathrm{~mL})$ and water $(10 \mathrm{~mL})$ were added. The organic layer was separated and the aqueous layer was extracted with ether $\left(3 \_10 \mathrm{~mL}\right)$. The organic layers were combined, dried over anh. $\mathrm{MgSO}_{4}$ and concentrated in vacuo. The residual oil was purified by column chromatography (deactivated silica gel, eluted with pet-ether : EtOAc $=10: 1$ ) to give the title compound as a clear oil $\left(76.0 \mathrm{mg}, 0.208 \mathrm{mmol}, 70 \%\right.$ yield), $\mathrm{R}_{\mathrm{f}} 0.50$ (petether : $\mathrm{EtOAc}=10: 1) .[\alpha]_{\mathrm{D}}+40.2\left(c 1.0, \mathrm{CHCl}_{3}\right)$.

IR (neat film): 3064, 2943, 2867, 1651, 1464, 1384, 1241, 1139, 1110, 1071, $1003 \mathrm{~cm}^{-1}$. ${ }^{1} \mathrm{H}$ NMR $\left(\mathrm{CDCl}_{3}, 500 \mathrm{MHz}\right): \delta 7.21 \sim 7.37(\mathrm{~m}, 10 \mathrm{H}), 6.31(\mathrm{~m}, 1 \mathrm{H}), 4.68(\mathrm{td}, J=5.7,2.1$ $\mathrm{Hz}, 1 \mathrm{H}), 4.63$ (d, $J=12.4 \mathrm{~Hz}, 1 \mathrm{H}), 4.60(\mathrm{~d}, J=11.4 \mathrm{~Hz}, 1 \mathrm{H}), 4.56(\mathrm{~d}, J=12.4 \mathrm{~Hz}, 1$ H), $4.42(\mathrm{~d}, J=11.4 \mathrm{~Hz}, 1 \mathrm{H}), 3.78$ (dd, $J=10.5,1.9 \mathrm{~Hz}, 1 \mathrm{H}), 3.69$ (dd, $J=10.5,4.9 \mathrm{~Hz}$, $1 \mathrm{H}$ ), 3.59 (ddd, $J=11.9,9.5,4.6 \mathrm{~Hz}, 1 \mathrm{H}$ ), 3.50 (ddd, $J=9.2,4.9,1.9 \mathrm{~Hz}, 1 \mathrm{H}$ ), 3.45 (m, $1 \mathrm{H}$ ), 3.43 (ddd, $J=9.5,9.3,5.3 \mathrm{~Hz}, 1 \mathrm{H}$ ), 2.63 (dt, $J=11.6,4.3 \mathrm{~Hz}, 1 \mathrm{H}$ ), 2.33 (ddd, $J=$ 16.4, 5.3, $1.2 \mathrm{~Hz}, 1 \mathrm{H}$ ), 2.09 (ddt, $J=16.4,9.3,2.1 \mathrm{~Hz}, 1 \mathrm{H}), 1.56$ (app q, $J \sim 11 \mathrm{~Hz}, 1$ H). ${ }^{13} \mathrm{C}$ NMR $\left(\mathrm{CDCl}_{3}, 125 \mathrm{MHz}\right): \delta$ 143.0, 138.2, 137.9, 128.4, 128.3, 127.9, 127.7, 127.6, 98.6, 80.3, 74.4, 73.5, 72.9, 72.2, 71.1, 69.2, 35.0, 26.8. HRMS Calcd for $\mathrm{C}_{23} \mathrm{H}_{26} \mathrm{O}_{4}\left(\mathrm{M}^{+}\right): 366.1831$. Found: 366.1836 . 
Preparation of $(4 \mathrm{a} S, 6 R, 7 S, 8 \mathrm{a} R)$-7-Benzyloxy-6-benzyloxymethyl-hexahydropyrano[3,2-b]-2-one (12):

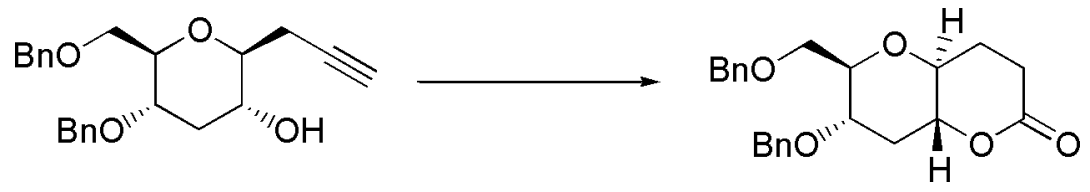

A mixture of alcohol 8 (56.2 mg, $0.153 \mathrm{mmol})$, complex $4 \mathbf{b}$ (13.8 mg, $0.0153 \mathrm{mmol})$, tris(p-methoxy)-phenylphosphine (21.6 mg, $0.0613 \mathrm{mmol}), \mathrm{N}$-hydroxysuccinimide (106 mg, $0.922 \mathrm{mmol})$, sodium bicarbonate $(25.7 \mathrm{mg}, 0.306 \mathrm{mmol})$, and tetra-n-butylammonium hexafluorophosphate $(17.8 \mathrm{mg}, 0.0459 \mathrm{mmol})$ in DMF $(0.38 \mathrm{~mL}, 0.4 \mathrm{M})$ were placed in a pre-heated oil bath at $85{ }^{\circ} \mathrm{C}$ and the solution was stirred for $20 \mathrm{~h}$. The reaction mixture was cooled to rt and purified by column chromatography (pet-ether : EtOAc $=1: 1$ ) without work-up to give the title compound as a white solid $(35 \mathrm{mg}, 0.092 \mathrm{mmol}, 60 \%$ yield), m.p $155-157^{\circ} \mathrm{C}$ (from pet-ether/ether); (lit ${ }^{4} 155-156^{\circ} \mathrm{C}$ ), $\mathrm{R}_{\mathrm{f}} 0.35$ (petether:EtOAc=1:1). $[\alpha]_{\mathrm{D}}+94.1\left(\mathrm{c} 0.52, \mathrm{CHCl}_{3}\right) ;\left(\right.$ lit $[\alpha]_{\mathrm{D}}+98.1\left(\right.$ c $\left.\left.0.61, \mathrm{CHCl}_{3}\right)\right)$. The spectral data are in full accord with literature values. ${ }^{4}$

IR (neat film): 3030, 2867, 1950, 1749, 1595, 1497, 1454, 1366, 1310, 1252, $1203 \mathrm{~cm}^{-1}$. ${ }^{1} \mathrm{H}$ NMR $\left(\mathrm{CDCl}_{3}, 500 \mathrm{MHz}\right): \delta$ 7.12-7.25 (m, $\left.10 \mathrm{H}\right), 4.59(\mathrm{~d}, J=11.2 \mathrm{~Hz}, 1 \mathrm{H}), 4.55(\mathrm{~d}, J$ $=11.4 \mathrm{~Hz}, 1 \mathrm{H}), 4.52(\mathrm{~d}, J=11.2 \mathrm{~Hz}, 1 \mathrm{H}), 4.39(\mathrm{~d}, J=11.4 \mathrm{~Hz}, 1 \mathrm{H}), 3.95(\mathrm{ddd}, J=$ 11.4, 9.4, 4.4 Hz, $1 \mathrm{H}), 3.73$ (dd, $J=10.7,1.7 \mathrm{~Hz}, 1 \mathrm{H}), 3.65(\mathrm{dd}, J=10.7,4.7 \mathrm{~Hz}, 1 \mathrm{H})$, $3.56(\mathrm{ddd}, J=10.8,9.4,4.6 \mathrm{~Hz}, 1 \mathrm{H}), 3.47(\mathrm{ddd}, J=9.6,4.7,1.7 \mathrm{~Hz}, 1 \mathrm{H}), 2.74(\mathrm{ddd}, J=$ 12.2, 8.9, $4.7 \mathrm{~Hz}, 1 \mathrm{H}), 2.66$ (app dt, $J=11.7,4.4 \mathrm{~Hz}, 1 \mathrm{H}), 2.58$ (dt, $J=17.9,8.2 \mathrm{~Hz}, 1$ $\mathrm{H}), 2.19(\mathrm{~m}, 1 \mathrm{H}), 1.88(\mathrm{~m}, 1 \mathrm{H}), 1.40(\mathrm{~m}, 1 \mathrm{H}) .{ }^{13} \mathrm{C} \mathrm{NMR}\left(\mathrm{CDCl}_{3}, 75 \mathrm{MHz}\right): \delta 170.3$, $137.9,137.6,128.4,128.3,127.9,127.8,127.7,80.5,77.2,75.7,74.4,73.5,71.5,71.4$, $68.8,35.3,27.9,24.6$.

\section{Preparation of 13}
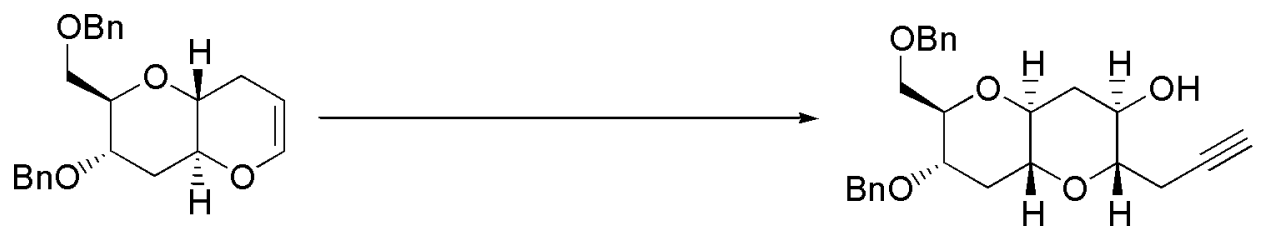

To a stirred solution of compound $11(130 \mathrm{mg}, 0.355 \mathrm{mmol})$ in $\mathrm{CH}_{2} \mathrm{Cl}_{2}(5 \mathrm{~mL})$ was added

${ }^{4}$ Kadota, I.; Takamura, H.; Sato, K.; Yamamoto, Y. J. Org. Chem. 2002, 67, 3494. 
freshly prepared dimethyldioxirane $(0.1 \mathrm{M}$ solution in acetone, $4 \mathrm{~mL})$ dropwise at $-78^{\circ} \mathrm{C}$ and the solution was stirred for $30 \mathrm{~min}$. The solution was concentrated in vacuo and the residual solvent was completely removed under high vacuum. The crude oil was dissolved in ether $(4 \mathrm{~mL})$ and cooled to $-78^{\circ} \mathrm{C}$. To this solution was added freshly prepared allenylmagnesium bromide $(1 \mathrm{M}$ solution in ether, $0.5 \mathrm{~mL})$ and the resulting solution was stirred for $2 \mathrm{~h}$. Water $(2 \mathrm{~mL})$ was added to this solution and the mixture was allowed to warm to $0^{\circ} \mathrm{C}$. Saturated aq. $\mathrm{NH}_{4} \mathrm{Cl}$ solution $(2 \mathrm{~mL})$ was added and the solution was vigorously stirred for $1 \mathrm{~h}$. The organic layer was separated and the aqueous layer was extracted with ether $\left(3_{-} 10 \mathrm{~mL}\right)$. The organic layers were combined, dried over anh. $\mathrm{MgSO}_{4}$ and concentrated in vacuo. The residual oil was purified by column chromatography (silica gel, eluted with pet-ether:EtOAc $=10: 1$ to $4: 1$ ) to give a mixture of isomers as a clear oil ( $94 \mathrm{mg}, 0.241 \mathrm{mmol}, 70 \%$ yield), which was taken up in the next reaction.

\section{Preparation of $(2 S, 4 a S, 6 R, 7 S, 8 a R)$-7-Benzyloxy-6-benzyloxymethyl-2-prop-2- ynyl-hexahydro-pyrano[3,2-b]pyran-3-one (14)}
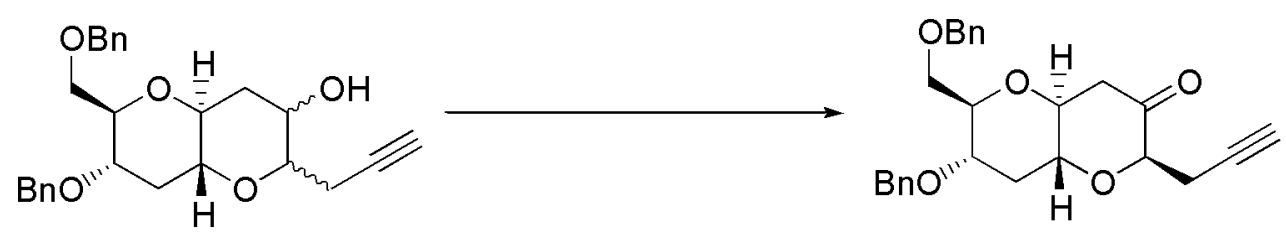

11:1

To a solution of alcohol 13 (90 mg, $0.213 \mathrm{mmol}$, mixtures of diastereomers) in $\mathrm{CH}_{2} \mathrm{Cl}_{2}$ $(3 \mathrm{~mL})$ was added pyridinium chlorochromate $(100 \mathrm{mg}, 0.425 \mathrm{mmol})$, molecular sieves $(4 \mathrm{~A}, 300 \mathrm{mg})$ and sodium acetate $(20 \mathrm{mg}, 0.042 \mathrm{mmol})$. After stirring at $\mathrm{rt}$ for $16 \mathrm{~h}$, ether $(10 \mathrm{~mL})$ was added to this solution and the mixture was stirred for $30 \mathrm{~min}$. The reaction mixture was filtered through a pad of celite and concentrated in vacuo. The residual oil was purified by column chromatography (silical gel, eluted with pet-ether:EtOAc=7:1) to give the title compound as a clear oil $(81 \mathrm{mg}, 0.194 \mathrm{mmol}, 91 \%$ yield, as a $11: 1$ mixture of diastereomers), $\mathrm{R}_{\mathrm{f}} 0.55$ (pet-ether:EtOAc $=4: 1$ ).

IR (neat film): v 3312, 2926, 2856, 2121, 1717, 1456, 1411, 1372, $1083 \mathrm{~cm}^{-1} .{ }^{1} \mathrm{H}$ NMR $\left(\mathrm{CDCl}_{3}, 500 \mathrm{MHz}\right): \delta$ 7.11-7.28 (m, $\left.10 \mathrm{H}\right), 4.63(\mathrm{~d}, J=12.3 \mathrm{~Hz}, 1 \mathrm{H}), 4.62(\mathrm{~d}, J=11.4$ $\mathrm{Hz}, 1 \mathrm{H}), 4.55(\mathrm{~d}, J=12.3 \mathrm{~Hz}, 1 \mathrm{H}), 4.42(\mathrm{~d}, J=11.4 \mathrm{~Hz}, 1 \mathrm{H}), 4.20(\mathrm{dd}, \mathrm{J}=7.8,5.2 \mathrm{~Hz}$, 
$1 \mathrm{H}), 3.76(\mathrm{dd}, J=10.6,1.7 \mathrm{~Hz}, 1 \mathrm{H}), 3.73(\mathrm{dd}, J=4.3,1.7 \mathrm{~Hz}, 1 \mathrm{H}), 3.63 \sim 3.71(\mathrm{~m}, 1 \mathrm{H})$, 3.45 (m, 2 H), 3.02 (dd, $J=7.0,5.6 \mathrm{~Hz}, 1 \mathrm{H}), 2.70$ (m, 2 H), 2.58 (m, 2 H), 2.09 (t, $J=$ $2.7 \mathrm{~Hz}, 1 \mathrm{H}), 1.58(\mathrm{~m}, 1 \mathrm{H}), 1.22$ (app q, $J=11.2 \mathrm{~Hz}, 1 \mathrm{H}) .{ }^{13} \mathrm{C} \mathrm{NMR}\left(\mathrm{CDCl}_{3}, 75 \mathrm{MHz}\right): \delta$ 206.2, 137.9, 137.7, 128.4, 128.4, 127.9, 128.8, 127.7, 127.6, 79.9, 79.2, 79.1, 75.4, 73.5, 72.0, 71.6, 71.1, 69.4, 68.8, 43.7, 35.2, 21.3. Anal. Calcd for $\mathrm{C}_{26} \mathrm{H}_{28} \mathrm{O}_{5}$ : C, 74.26; H, 6.71. Found: C, 74. 42; H, 6.82 .

Preparation of ( $2 S, 3 R, 4 a S, 6 R, 7 S, 8 a R)$-7-Benzyloxy-6-benzyloxymethyl-2-prop-2ynyl-octahydro-pyrano[3,2-b]pyran-3-ol (15) :

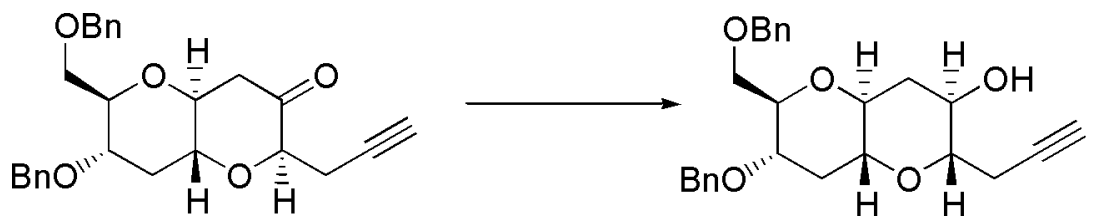

A mixture of ketone $14(37 \mathrm{mg}, 0.088 \mathrm{mmol})$ was dissolved in toluene $(1 \mathrm{~mL})$ and diazabicycloundecene $(1.3 \mathrm{mg}, 0.0088 \mathrm{mmol})$ was added. After stirring at $80^{\circ} \mathrm{C}$ for $16 \mathrm{~h}$, the reaction mixture was cooled to $\mathrm{rt}$ and purified by column chromatography without extractive work-up to give ( $2 R, 4 \mathrm{a} S, 6 R, 7 S, 8 \mathrm{a} R)$-7-Benzyloxy-6-benzyloxymethyl-2prop-2-ynyl-hexahydro-pyrano[3,2-b]pyran-3-one (20:1 mixture) as a clear oil (33 $\mathrm{mg}, 0.079 \mathrm{mmol}, 88 \%$ yield), $\mathrm{R}_{\mathrm{f}} 0.55$ (pet-ether:EtOAc $\left.=4: 1\right)$. $[\alpha]_{\mathrm{D}} 50.4\left(c 1.11, \mathrm{CHCl}_{3}\right)$. IR (neat film): v 3293, 2939, 2360, 1712, 1449, 1428, $1130 \mathrm{~cm}^{-1} .{ }^{1} \mathrm{H}$ NMR $\left(\mathrm{CDCl}_{3}, 300\right.$ MHz): $\delta$ 7.21-7.37 (m, $10 \mathrm{H}), 4.63$ (d, $J=12.2 \mathrm{~Hz}, 1 \mathrm{H}), 4.60(\mathrm{~d}, J=11.3 \mathrm{~Hz}, 1 \mathrm{H}), 4.54$ (d, $J=12.2 \mathrm{~Hz}, 1 \mathrm{H}), 4.41$ (d, $J=11.3 \mathrm{~Hz}, 1 \mathrm{H}), 3.94$ (dd, $J=7.2,4.2 \mathrm{~Hz}, 1 \mathrm{H}), 3.76$ (dd, $J=10.7,1.9 \mathrm{~Hz}, 1 \mathrm{H}), 3.69(\mathrm{dd}, J=10.7 \mathrm{~Hz}, 4.6 \mathrm{~Hz}, 1 \mathrm{H}), 3.64$ (ddd, $J=11.1,9.4,4.6$ $\mathrm{Hz}, 1 \mathrm{H}$ ), 3.48 (ddd, $J=9.4,4.6,1.9 \mathrm{~Hz}, 1 \mathrm{H}), 3.40-3.45$ (m, $2 \mathrm{H}$ ), 3.00 (dd, $J=15.6,4.9$ $\mathrm{Hz}, 1 \mathrm{H}), 2.79$ (ddd, $J=17.4,4.2,2.7 \mathrm{~Hz}, 1 \mathrm{H}), 2.74$ (m, $1 \mathrm{H}), 2.54$ (dd, $J=15.6,11.4 \mathrm{~Hz}$, $1 \mathrm{H}), 2.50(\mathrm{ddd}, J=17.4,7.2,2.7 \mathrm{~Hz}, 1 \mathrm{H}), 2.01(\mathrm{t}, J=2.6 \mathrm{~Hz}, 1 \mathrm{H}), 1.63(\mathrm{~m}, 1 \mathrm{H}) .{ }^{13} \mathrm{C}$ NMR $\left(\mathrm{CDCl}_{3}, 75 \mathrm{MHz}\right): \delta 203.3,137.9,137.7,128.4,28.3,127.9,127.8,127.7,127.6$, 80.9, 80.2, 79.9, 76.3, 75.1, 73.5, 71.8, 71.1, 70.0, 68.8, 44.8, 34.9, 19.8. HRMS Calcd for $\mathrm{C}_{26} \mathrm{H}_{28} \mathrm{O}_{5}: 420.1937$. Found: 420.1944.

To a stirred solution of this ketone $(49 \mathrm{mg}, 0.117 \mathrm{mmol})$ in THF $(1.5 \mathrm{~mL})$ was added lithium aluminum hydride $(4.4 \mathrm{mg}, 0.117 \mathrm{mmol})$ at $0^{\circ} \mathrm{C}$ and the solution was stirred for $30 \mathrm{~min}$. Excess sodium sulfate decahyderate ( $\sim 60 \mathrm{mg}$ ) was added and the solution was 
stirred for an additional $30 \mathrm{~min}$. The solution was filtered through a pad of celite and concentrated in vacuo. The residual oil was purified by column chromatography (silica gel, eluted with pet-ether;EtOAc $=10: 1)$ to give the title compound $\mathbf{1 5}$ as a clear oil consisting of 13:1 diastereomeric mixture ( $47 \mathrm{mg}, 0.112 \mathrm{mmol}, 95 \%$ yield), $\mathrm{R}_{\mathrm{f}} 0.35$ (petether:EtOAc $=2: 1)$. $[\alpha]_{\mathrm{D}} 65.5\left(\mathrm{c} 1.11, \mathrm{CHCl}_{3}\right)$.

IR (neat film): $v$ 3445, 3289, 2936, 2875, 1442, 1371, 1282, 1274, 1165, $989 \mathrm{~cm}^{-1} .{ }^{1} \mathrm{H}$ NMR $\left(\mathrm{CDCl}_{3}, 300 \mathrm{MHz}\right): \delta 7.19 \sim 7.37(\mathrm{~m}, 10 \mathrm{H}), 4.62(\mathrm{~d}, J=12.2 \mathrm{~Hz}, 1 \mathrm{H}), 4.56(\mathrm{~d}, J=$ $11.4 \mathrm{~Hz}, 1 \mathrm{H}), 4.55$ (d, $J=12.2 \mathrm{~Hz}, 1 \mathrm{H}), 4.37$ (d, $J=11.4 \mathrm{~Hz}, 1 \mathrm{H}), 3.75(\mathrm{dd}, J=10.6$, $1.8 \mathrm{~Hz}, 1 \mathrm{H}), 3.64$ (dd, $J=10.6,5.0 \mathrm{~Hz}, 1 \mathrm{H}), 3.62$ (m, $1 \mathrm{H}), 3.52$ (ddd, $J=11.0,9.5,4.4$ $\mathrm{Hz}, 1 \mathrm{H}), 3.44$ (ddd, $J=9.4,5.0,1.8 \mathrm{~Hz}, 1 \mathrm{H}), 3.26$ (ddd, $J=9.4,5.9,4.5 \mathrm{~Hz}, 1 \mathrm{H}), 3.10$ (ddd, $J=11.4,9.0,4.2 \mathrm{~Hz}, 1 \mathrm{H}), 3.06(\mathrm{ddd}, J=11.4,9.0,4.0 \mathrm{~Hz}, 1 \mathrm{H}), 2.65$ (ddd, $J=$ 17.2, 4.5, $2.8 \mathrm{~Hz}, 1 \mathrm{H}$ ), 2.57 (ddd, $J=11.0,4.4,4.0 \mathrm{~Hz}, 1 \mathrm{H}$ ), 2.56 (ddd, $J=17.2,5.9,2.8$ Hz, $1 \mathrm{H}$ ), 2.45 (ddd, $J=11.4,4.4,4.2 \mathrm{~Hz}, 1 \mathrm{H}), 2.06$ (t, $J=2.8 \mathrm{~Hz}, 1 \mathrm{H}), 1.54$ (app q, $J=$ $11.0 \mathrm{~Hz}, 1 \mathrm{H}), 1.49$ (app q, $J=11.0 \mathrm{~Hz}, 1 \mathrm{H}) .{ }^{13} \mathrm{C} \mathrm{NMR}\left(\mathrm{CDCl}_{3}, 75 \mathrm{MHz}\right): \delta 138.3,138.0$, 128.3, 128.2, 127.8, 127.7, 127.5, 80.9, 79.6, 78.4, 77.2, 73.6, 73.5, 72.3, 71.6, 70.6, 69.3, 69.2, 68.3, 35.6, 32.2, 20.2. HRMS Calcd for $\mathrm{C}_{26} \mathrm{H}_{30} \mathrm{O}_{5}\left(\mathrm{M}^{+}\right)$: 422.2093. Found: 422.2098 .

\section{One-pot procedure for the synthesis of the alcohol 13 from 11 :}

Ketone $11(42 \mathrm{mg}, 0.10 \mathrm{mmol})$ was dissolved in toluene $(1 \mathrm{~mL})$ and diazabicycloundecene $\left(1.5 \mathrm{mg}, 0.010 \mathrm{mmol}\right.$ ) was added. Then the solution was stirred at $80^{\circ} \mathrm{C}$ for $24 \mathrm{~h}$. The reaction mixture was concentrated and re-dissolved in THF $(1.5 \mathrm{~mL})$. To the resulting solution was added lithium aluminum hydride $(3.8 \mathrm{mg}, 0.100 \mathrm{mmol})$ at $0^{\circ} \mathrm{C}$ and the solution was stirred at that temperature for $1 \mathrm{~h}$. Excess sodium sulfate decahydrate was added ( $\sim 100 \mathrm{mg}$ ) and the solution was stirred for an additional $1 \mathrm{~h}$. The solution was filtered through a pad of celite and concentrated in vacuo. The residual oil was purified by column chromatography (silica gel, eluted with pet-ether:EtOAc $=10: 1$ ) to give the title compound ( $36 \mathrm{mg}, 0.085 \mathrm{mmol}$ ) as clear oil ( $85 \%$ yield). The spectral data are in full accord with those previously obtained. 
Preparation of $(2 R, 3 S, 4 a R, 8 \mathrm{a} R, 9 \mathrm{a} S, 10 \mathrm{a} S)$-3-Benzyloxy-2-benzyloxymethyl3,4,4a,5,8a,9,9a, 10a-octahydro-2H-1,8,10-trioxa-anthracene (16)<smiles>C#CC[C@H]1O[C@H]2C[C@@H](O)C(COCc3ccccc3)O[C@H]2C[C@H]1O</smiles>

A mixture of alcohol 15 (42 $\mathrm{mg}, 0.100 \mathrm{mmol})$, Ru catalyst (4.2 mg, $0.0050 \mathrm{mmol})$, tris(p-fluorophenyl)phosphine $(6.2 \mathrm{mg}, 0.02 \mathrm{mmol}), \mathrm{N}$-hydroxysuccinimide sodium salt (6.9 $\mathrm{mg}, 0.050 \mathrm{mmol})$, tetra-n-butylammonium hexafluorophosphate $(5.8 \mathrm{mg}, 0.015 \mathrm{mmol})$ in DMF $(0.25 \mathrm{~mL}, 0.4 \mathrm{M})$ were placed in a pre-heated oil bath at $85^{\circ} \mathrm{C}$ and the solution was stirred at that temperature for $16 \mathrm{~h}$. The reaction mixture was cooled to $\mathrm{rt}$ and purified without aqueous work-up by column chromatography (deactivated silica gel, eluted with pet-ether:EtOAc $=4: 1)$ to give the pure compound 16 as a white solid (28 $\mathrm{mg}, 0.065$ mmmol, $65 \%$ yield), m.p $112-115^{\circ} \mathrm{C}$ (from pet-ether/ether), $\mathrm{R}_{\mathrm{f}} 0.45$ (pet-ether:EtOAc $=$ 9:1). $[\alpha]_{\mathrm{D}}+66.3\left(c 1.11, \mathrm{CHCl}_{3}\right)$.

IR (neat film): v 2926, 2855, 1640, 1465, 1378, 1268, 1140, $1056 \mathrm{~cm}^{-1} .{ }^{1} \mathrm{H} \mathrm{NMR}\left(\mathrm{CDCl}_{3}\right.$, 300MHz): $\delta 7.21-7.39(\mathrm{~m}, 10 \mathrm{H}), 6.32(\mathrm{~m}, 1 \mathrm{H}), 4.68(\mathrm{~m}, 1 \mathrm{H}), 4.63$ (d, J=12.4 Hz, 1 H), $4.57(\mathrm{~d}, J=11.4 \mathrm{~Hz}, 1 \mathrm{H}), 4.39(\mathrm{~d}, J=11.4 \mathrm{~Hz}, 1 \mathrm{H}), 3.76(\mathrm{dd}, J=10.5,1.7 \mathrm{~Hz}, 1 \mathrm{H})$, $3.66(\mathrm{dd}, J=10.5,4.8 \mathrm{~Hz}, 1 \mathrm{H}), 3.57$ (ddd, $J=11.0,9.5,4.6 \mathrm{~Hz}, 1 \mathrm{H}), 3.50$ (ddd, $J=11.0$, 9.4, 4.6 Hz, 1H), 3.47 (ddd, $J=9.4,4.8,1.7 \mathrm{~Hz}, 1 \mathrm{H}), 3.43$ (m, $2 \mathrm{H}), 3.18$ (m, $2 \mathrm{H}), 2.62$ $(\mathrm{dt}, J=11.1,4.6 \mathrm{~Hz}, 1 \mathrm{H}), 2.56(\mathrm{dt}, J=11.3,4.5 \mathrm{~Hz}, 1 \mathrm{H}), 2.25$ (m, $1 \mathrm{H}), 2.04$ (ddt, J = $16.3,9.5,2.1 \mathrm{~Hz}, 1 \mathrm{H}), 1.63(\operatorname{app~q}, J=11.3 \mathrm{~Hz}, 1 \mathrm{H}), 1.50(\operatorname{app~q}, J=11.0 \mathrm{~Hz}, 1 \mathrm{H}) .{ }^{13} \mathrm{C}$ NMR $\left(\mathrm{CDCl}_{3}, 75 \mathrm{MHz}\right): \delta 143.2,138.1,137.8,128.4,128.3,127.9,127.8,127.6,98.5$, 80.3, 76.2, 75.9, 74.6, 73.5, 73.4, 72.2, 71.0, 69.0, 35.1, 34.9. 26.9. HRMS Calcd for $\mathrm{C}_{26} \mathrm{H}_{30} \mathrm{O}_{5}\left(\mathrm{M}^{+}\right):$422.2093. Found: 422.2090.

Preparation of $((2 R, 3 S, 4 \mathrm{a} R, 6 R, 8 \mathrm{a} S)-3$-Benzyloxy-2-benzyloxymethyl-6trimethylsilylethynyl-octahydropyrano[3,2-b]pyran (17)

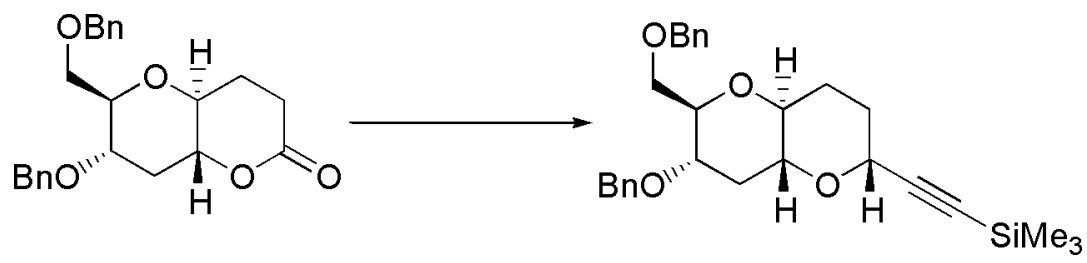


To a stirred solution of trimethylsilylacetylene $(192 \mathrm{mg}, 0.20 \mathrm{mmol})$ in THF $(1 \mathrm{~mL})$ was added $\mathrm{n}-\mathrm{BuLi}(1.6 \mathrm{M}$ solution in hexane, $0.1 \mathrm{~mL}, 0.16 \mathrm{mmol})$ at $-78^{\circ} \mathrm{C}$ dropwise and the solution was stirred for $1 \mathrm{~h}$. To this solution was added a solution of lactone 12 (38 mg, $0.10 \mathrm{mmol})$ in THF $(1 \mathrm{~mL})$ dropwise at $-78^{\circ} \mathrm{C}$. After stirring for $1 \mathrm{~h}$, aqueous $1 \mathrm{~N} \mathrm{HCl}(1$ $\mathrm{mL}$ ) was slowly added and the solution was allowed to warm to rt. The organic phase was separated and the aqueous layer was extracted with ether $\left(2_{-} 20 \mathrm{~mL}\right)$. The organic layers were combined, dried over anh. $\mathrm{MgSO}_{4}$, filtered thorough a pad of silica gel and concentrated in vacuo to give the title compound $\left(45 \mathrm{mg}\right.$ ) as a clear oil, $\mathrm{R}_{\mathrm{f}} 0.55$ (petether:EtOAc $=4: 1$ ). This compound was used for the next transformation without further purification.

IR (neat film): v 3320, 3031, 2954, 2868, 2178, 1544, 1434, 1361, 1250, 1184, $1071 \mathrm{~cm}^{-}$ 1. ${ }^{1} \mathrm{H}$ NMR (CDCl $\left.3,500 \mathrm{MHz}\right): \delta$ 7.25-7.35 (m, $\left.10 \mathrm{H}\right), 4.54(\mathrm{~d}, J=11.5 \mathrm{~Hz}, 1 \mathrm{H}), 4.52(\mathrm{~d}$, $J=12.3 \mathrm{~Hz}, 1 \mathrm{H}), 4.31(\mathrm{~d}, J=12.3 \mathrm{~Hz}, 1 \mathrm{H}), 4.37(\mathrm{~d}, J=11.5 \mathrm{~Hz}, 1 \mathrm{H}), 3.75(\mathrm{~m}, 2 \mathrm{H})$, 3.62 (m, 2 H), 3.25-3.40 (m, 2 H), 1.82 (m, 2 H), 1.25-1.40 (m, 4 H), 0.15, 0.08 (s, 9 H).

To a stirred solution of this alcohol in $\mathrm{CH}_{3} \mathrm{CN}(0.5 \mathrm{~mL})$ and $\mathrm{CH}_{2} \mathrm{Cl}_{2}(0.5 \mathrm{~mL})$ at $-40^{\circ} \mathrm{C}$ was added a solution of triethylsilane $(48 \mathrm{mg}, 0.30 \mathrm{mmol}$ ) and boron trifluoride etherate (43 mg, $0.30 \mathrm{mmol})$ in $\mathrm{CH}_{3} \mathrm{CN}(0.5 \mathrm{~mL})$ and $\mathrm{CH}_{2} \mathrm{Cl}_{2}(0.5 \mathrm{~mL})$ at $-40^{\circ} \mathrm{C}$ via cannula. After stirring at $-40^{\circ} \mathrm{C}$ for $4 \mathrm{~h}$, sat. $\mathrm{NaHCO}_{3}$ solution $(1 \mathrm{~mL})$ was added, and the reaction mixture was allowed to warm to rt. The organic phase was separated and the aqueous layer was extracted with $\mathrm{CH}_{2} \mathrm{Cl}_{2}(2-5 \mathrm{~mL})$. The organic layers were combined, dried over anh. $\mathrm{MgSO}_{4}$ and concentrated in vacuo. The residual oil was purified by column chromatography (silica gel, eluted with pet-ether:EtOAc $=10: 1$ ) to give the title compound as a clear oil $(73 \%$ yield, $34 \mathrm{mg}, 0.073 \mathrm{mmol}), \mathrm{R}_{\mathrm{f}} 0.55$ (pet-ether:EtOAc $=$ 9:1). $[\alpha]_{\mathrm{D}}+91.3\left(c 1.11, \mathrm{CHCl}_{3}\right)$.

IR (neat film): v 3029, 2930, 2860, 1651, 1496, 1454, 1337, 1236, 1110, $1056 \mathrm{~cm}^{-1} .{ }^{1} \mathrm{H}$ NMR ( $\left.\mathrm{CDCl}_{3}, 500 \mathrm{MHz}\right): \delta 7.25-7.35(\mathrm{~m}, 10 \mathrm{H}), 4.31$ (d, $\left.J=12.3 \mathrm{~Hz}, 1 \mathrm{H}\right), 4.54$ (d, $J=$ $11.5 \mathrm{~Hz}, 1 \mathrm{H}), 4.52$ (d, $J=12.3 \mathrm{~Hz}, 1 \mathrm{H}), 4.37$ (d, $J=11.5 \mathrm{~Hz}, 1 \mathrm{H}), 4.13$ (dd, $J=11.5$ $\mathrm{Hz}, 2.3 \mathrm{~Hz}, 1 \mathrm{H}), 3.74$ (dd, $J=10.6,1.7 \mathrm{~Hz}, 1 \mathrm{H}), 3.64$ (dd, $J=10.6,4.9 \mathrm{~Hz}, 1 \mathrm{H}), 3.51$ (ddd, $J=10.6,9.6,4.6 \mathrm{~Hz}, 1 \mathrm{H}), 3.43$ (ddd, $J=9.4,4.9,1.7 \mathrm{~Hz}, 1 \mathrm{H}), 3.06(\mathrm{~m}, 2 \mathrm{H}), 2.53$ $(\mathrm{dt}, J=11.4,4.3 \mathrm{~Hz}, 1 \mathrm{H}), 2.10(\mathrm{~m}, 1 \mathrm{H}), 1.99(\mathrm{~m}, 1 \mathrm{H}), 1.81(\mathrm{~m}, 1 \mathrm{H}), 1.39(\mathrm{~m}, 2 \mathrm{H})$, 0.16 (s, $9 \mathrm{H}) .{ }^{13} \mathrm{C} \mathrm{NMR}\left(\mathrm{CDCl}_{3}, 125 \mathrm{MHz}\right): \delta 138.2,137.9,128.4,128.3,127.9,127.8$, 
127.7, 127.6, 103.4, 89.6, 80.3, 76.7, 76.5, 73.4, 72.4, 71.0, 69.2, 68.2, 35.4, 32.0, 29.0, 0.2. Anal.Calcd for $\mathrm{C}_{28} \mathrm{H}_{36} \mathrm{O}_{4} \mathrm{Si}$ : C, 72.37; H, 7.81. Found: C, 72.39; H, 7.88. 